\title{
Uretrostomia pré-púbica videoassistida em um felino com estenose uretral
}

\section{Laparoscopic-assisted prepubic urethrostomy in a cat with urethral stenosis}

\author{
Saulo Tadeu Lemos Pinto Filho ${ }^{1}$; Marilia Teresa Oliveira ${ }^{1}$; Fernando Wiecheteck \\ Souza $^{1}$; Fabíola Dalmolin ${ }^{1}$; Hellen Hartmann²; Antônio Soares Coutinho Júnior ${ }^{1}$; \\ Lucas Antonio Heinen Schuster ${ }^{2}$; Carlos Afonso Castro Beck ${ }^{3}$; Felipe Ribeiro \\ Botelho Santos ${ }^{2}$; João Pedro Scussel Feranti ${ }^{1}$; Mauricio Veloso Brun ${ }^{4 *}$
}

\section{Resumo}

\begin{abstract}
A uretrostomia é técnica cirúrgica utilizada em casos de obstrução parcial ou total da uretra, e na abordagem laparoscópica pode ser uma opção de tratamento. Devido à escassez de estudos envolvendo essa técnica, este trabalho relata o caso de uretrostomia pré-púbica videoassistida (laparoscópica híbrida) em um felino com estenose uretral. O paciente de cinco anos de idade apresentando histórico de doença do trato urinário inferior dos felinos (DTUIF) recorrente, havia sido submetido a uretrostomia perineal há dois anos e nove meses. Depois desse procedimento, o paciente passou por mais quatro cirurgias corretivas sem sucesso. Após realização de exames laboratoriais (hemograma e bioquímicos) e de imagem, o animal foi submetido a uretrostomia pré-púbica laparoscópica híbrida, obtendo-se sucesso terapêutico e possibilitando ao paciente adequada qualidade de vida. Conclui-se que esta técnica é viável e segura em gatos, tornando-a uma possível escolha de tratamento cirúrgico para estenose uretral. Palavras-chave: Estenose da uretra, videocirurgia, gatos, cirurgia urológica
\end{abstract}

\begin{abstract}
Urethrostomy is a surgical technique used in cases of partial or total obstruction of the urethra, and the laparoscopic approach may be a treatment option. Due to the scarcity of studies involving the technique, this paper reports the case of laparoscopic-assisted pre-pubic urethrostomy (hybrid laparoscopic) in a cat with urethral stricture. A five-years-old cat presenting recurrent feline lower urinary tract disease (FLUTD) had undergone to perineal urethrostomy two years and nine months ago. After this procedure, the patient underwent another four corrective surgeries without success. Laboratory tests (blood count and biochemical) and image diagnosis were realized. The patient was subjected to laparoscopic-assisted pre-pubic urethrostomy and it was achieved therapeutic success and adequate patient quality of life. It is concluded that this technique is feasible and safe in cats, making it a possible choice of surgical treatment for urethral stricture.
\end{abstract}

Key words: Urethral stricture, videosurgery, cats, urologic surgery

\footnotetext{
${ }^{1}$ Discentes do Programa de Pós-graduação em Medicina Veterinária da Universidade Federal de Santa Maria, UFSM, Santa Maria, RS.E-mail: saulovet2011@hotmail.com; marilia2805@hotmail.com; wiecheteck@hotmail.com; fabioladalmolin@hotmail.com; antonioscj@gmail.com; johny.sf@hotmail.com

${ }^{2}$ Residentes do Hospital Veterinário, UFSM, Santa Maria, RS. E-mail: hellenhartmann@gmail.com; lucaschuster@hotmail.com; feliperbsantos@hotmail.com

${ }^{3}$ Prof. da Faculdade de Medicina Veterinária da Universidade Federal do Rio Grande do Sul, UFRGS, Porto Alegre, RS. E-mail: afonso.beck@ibest.com.br

${ }^{4}$ Médico Veterinário, Prof. do Dept ${ }^{\mathrm{o}}$ de Clínica de Pequenos Animais, UFSM, Santa Maria, RS. E-mail: mauriciovelosobrun@, hotmail.com

* Autor para correspondência
} 


\section{Introdução}

Doença do trato urinário inferior dos felinos (DTUIF) é um termo utilizado para descrever distúrbios de gatos domésticos, vesicopatias e uretropatias, clinicamente caracterizados por hematúria, disúria, polaciúria e obstrução uretral parcial ou completa (ROSA; QUITZAN, 2011). O tratamento da obstrução uretral deve ser emergencial devido ao risco de óbito, e baseia-se no alívio da obstrução, correção dos efeitos sistêmicos da uremia e na prevenção de sua recidiva (GALVÃO et al., 2010).

A uretrostomia pré-púbica é indicada em obstruções graves na uretra, processos de estenose, além de traumatismos irreparáveis. A emissão de urina volta a normalidade dentro de 24 horas no pós-operatório (KAUFMANN; NEVES; HABERMANN, 2009). Em um estudo retrospectivo foram investigados as indicações e resultados em longo prazo e, os autores concluíram que este é um procedimento de salvamento, o qual pode aliviar os sinais de obstrução do trato urinário e evitar o agravamento da obstrução. No entanto, as complicações potenciais devem limitar seu uso a casos em que as técnicas padrão para uretrostomia perineal não podem ser executadas (BAINES; RENNIE; WHITE, 2001). A uretrostomia prépúbica predispõe a infecção do trato urinário devido à redução no comprimento da uretra e aumento do seu diâmetro. O risco de estenose é mínimo, pois nesta região a uretra é de 3 a 4 vezes maior que na perineal. A menos que ocorram danos no esfíncter e a fonte vascular da junção vesicouretral, a maior parte dos animais permanece continente após esse procedimento (PINTO, 2011; BERNARDE; VIGUIER, 2004). A falta de tensão tecidual no orifício uretral e a justaposição da mucosa uretral a margem da pele são essenciais para impedir a dermatite induzida pelo contato da urina (PINTO, 2011; LEÓN et al., 1997).

Considerando não haver nenhum relato de utilização da cirurgia videoassistida (laparoscópica híbrida) na realização da uretrostomia pré-púbica em gatos, foi executado um experimento em coelhos Nova Zelândia objetivando desenvolver essa técnica para futura aplicação na espécie referida. Oito animais foram operados e avaliados durante 30 dias após o procedimento cirúrgico. $\mathrm{O}$ autor concluiu que a uretrostomia pré-púbica auxiliada por laparoscopia é exequível, propondo ser testada futuramente no tratamento de gatos com problemas na uretra distal (PINTO, 2011).

Em virtude da escassez de estudos envolvendo essa técnica, o presente trabalho objetiva relatar um caso de uretrostomia pré-púbica laparoscópica híbrida em um felino com estenose uretral.

\section{Relato do Caso}

Foi atendido no hospital veterinário universitário da Universidade Federal de Santa Maria (UFSM) um felino, macho, SRD, de cinco anos de idade, que apresentava histórico de DTUIF recorrente. $\mathrm{O}$ animal havia sido submetido à primeira uretrostomia perineal há dois anos e nove meses; o procedimento resultou em estenose da uretra, o que proporcionou intervenções subsequentes para desobstrução, incluindo a realização de quatro cirurgias adicionais. A proprietária também relatou a presença de cistite recorrente. Ao exame clínico o animal apresentava temperatura de $38,6^{\circ} \mathrm{C}$, mucosas rosadas, desidratação estimada em $5 \%$ e, a palpação, distensão vesical. Nos primeiros exames laboratoriais realizados não foram detectadas alterações significativas. No exameultrassonográfico evidenciou-se bexiga moderadamente distendida, com conteúdo heterogêneo, parede regular e espessada; os rins apresentavam-se simétricos, com córtex espessado e discreta perda da diferenciação córtico-medular; e os ureteres estavam dilatados com conteúdo hipoecóico.

Passados quatro dias, os exames laboratoriais foram repetidos e, na ocasião, observou-se elevação nos níveis de ureia $(66,31 \mathrm{mg} / \mathrm{dL})$ e creatinina $(1,94 \mathrm{mg} / \mathrm{dL})$. Após esta avaliação, o animal foi 
encaminhado para urestrostomia laparoscópica híbrida pré-púbica. Para a realização da cirurgia, o felino foi pré-medicado com tramadol (2mg. $\mathrm{kg}^{-1}$, IM), e induzido com propofol (6mg.kg1, IV). A manutenção da anestesia foi realizada com isoflurano vaporizado em oxigênio a $100 \%$, enquanto a anestesia epidural constou da associação de lidocaína $\left(0,13 \mathrm{ml} . \mathrm{kg}^{-1}\right)$, bupivacaína $(0,13 \mathrm{ml}$. $\left.\mathrm{kg}^{-1}\right)$ e morfina $\left(0,1 \mathrm{mg} \cdot \mathrm{kg}^{-1}\right)$. Após a estabilização do plano anestésico, o paciente foi posicionado em decúbito dorsal e foi realizada cistocentese, pois não foi possível esvaziar a bexiga por meio de compressão ou sondagem uretral, em virtude do grau da estenose.

A uretrostomia foi realizada por meio de três portais de acesso $\left(\right.$ Storz $\left.^{\circledR}\right)$ em triangulação. O primeiro portal foi posicionado na cicatriz umbilical (5mm) pela técnica aberta, o segundo na região hipogástrica direita $(3 \mathrm{~mm})$ e o terceiro $(3 \mathrm{~mm})$ foi introduzido na região hipogástrica esquerda (Figura
1A). Mantendo-se o pneumoperitônio com $\mathrm{CO}_{2}(12$ $\mathrm{mmHg} ; 11 / \mathrm{min}$.), foi possível realizar a secção do ligamento mediano da bexiga e posterior dissecção uretral do colo vesical (Figura 1B), preservandose ao máximo a irrigação e inervação colateral. A uretra foi pinçada com Maryland (Storz ${ }^{\circledR}$ ) de $3 \mathrm{~mm}$ e exteriorizada através de um acesso pré-púbico convencional de aproximadamente $1,2 \mathrm{~cm}$ para a realização de sutura de reparo próximo a próstata (Figura 1C), expondo-se assim a uretra pélvica. Foi então realizada uretrotomia para sondagem uretral pela técnica convencional via sutura mucocutânea com polidioxanona 5-0 em padrão isolado simples (Figura 1B). A sutura da parede abdominal do primeiro portal $(5 \mathrm{~mm})$ foi realizada com pontos tipo sultan, utilizando ácido poliglicólico 2-0; o mesmo fio e padrão de sutura foram utilizados na sutura do tecido subcutâneo. Nos portais de $3 \mathrm{~mm}$ foi aplicada apenas sutura de pele, em padrão interrompido simples com mononylon 5-0. A ferida cutânea do portal de $5 \mathrm{~mm}$ também foi ocluída de forma similar.

Figura 1. (A) Imagem do posicionamento dos portais para realização da uretrostomia pré-púbica em um felino, macho, com cinco anos com obstrução uretral recorrente com estenose grave. (B) Imagem videolaparoscópica da uretra (seta) após dissecação. (C) Imagem videolaparoscópica da apreensão uretral com pinça de Maryland para posterior exteriorização em acesso pré-púbico. (D) Imagem fotográfica da realização da sondagem uretral após a etapa convencional da uretrostomia.

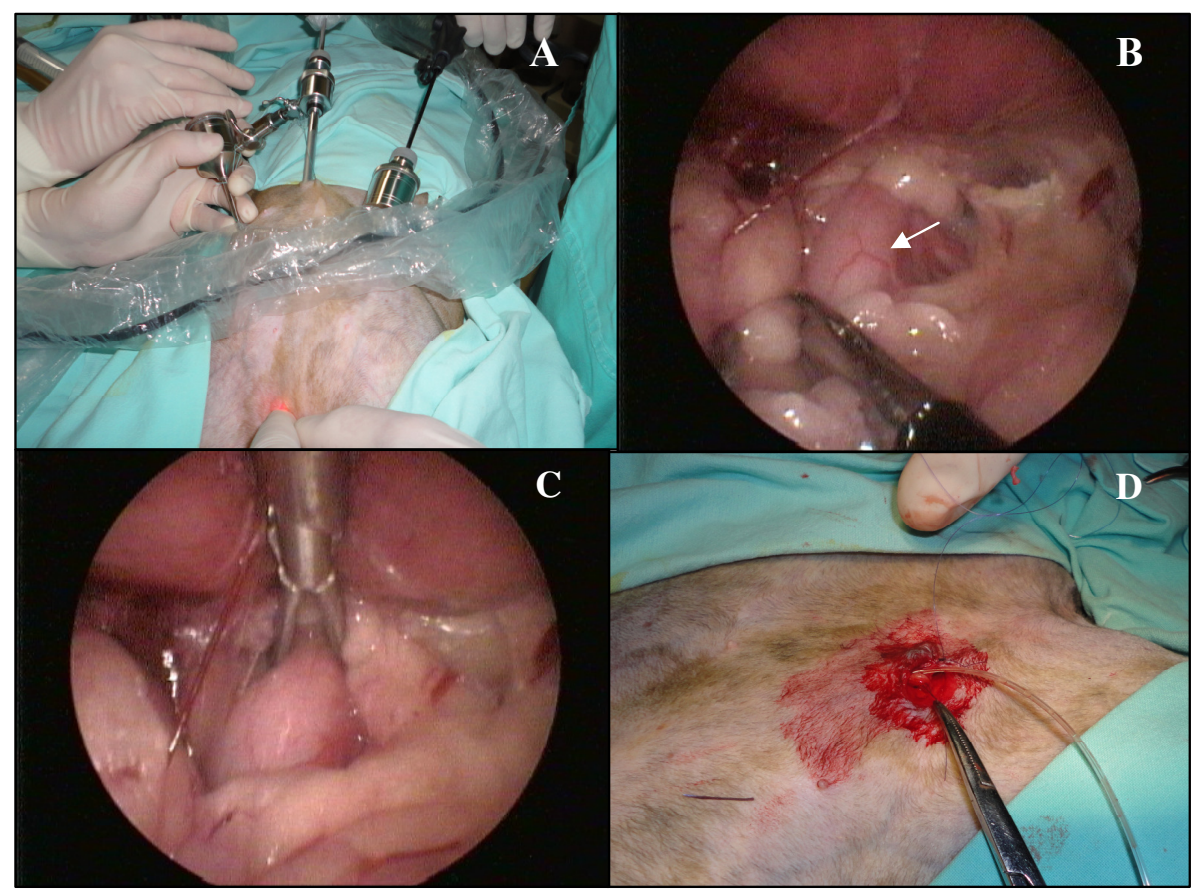

Fonte: Elaboração dos autores. 
No pós-operatório foram administrados cloridrato de tramadol $\left(2,5 \mathrm{mg} \cdot \mathrm{kg}^{-1}, \mathrm{IV}\right.$, TID, durante 2 dias), enrofloxacina (5mg. $\mathrm{kg}^{-1}, \mathrm{IV}, \mathrm{BID}$, sete dias) e associação de dipirona e brometo de hioscina (25mg. $\mathrm{kg}^{-1}$, SC, BID, dois dias). O paciente permaneceu internado nas primeiras 24 horas de pós-operatório com colar elizabetano, urinando espontaneamente. A sutura foi removida em 10 dias, sem a ocorrência de deiscência. Trinta dias após a cirurgia a proprietária retornou ao hospital com o animal para reavaliação do procedimento, sendo que o exame clínico demonstrou bom estado geral e ferida cirúrgica cicatrizada e sem presença de infecção (Figura 2).

Figura 2. (A) Imagem mostrando o aspecto da ferida cirúrgica trinta dias após a cirurgia de uretrostomia pré-púbica laparoscópica híbrida em um felino macho, cinco anos. Observar ferida cicatrizada e sem presença de infecção. (B) Detalhe da figura 2A.

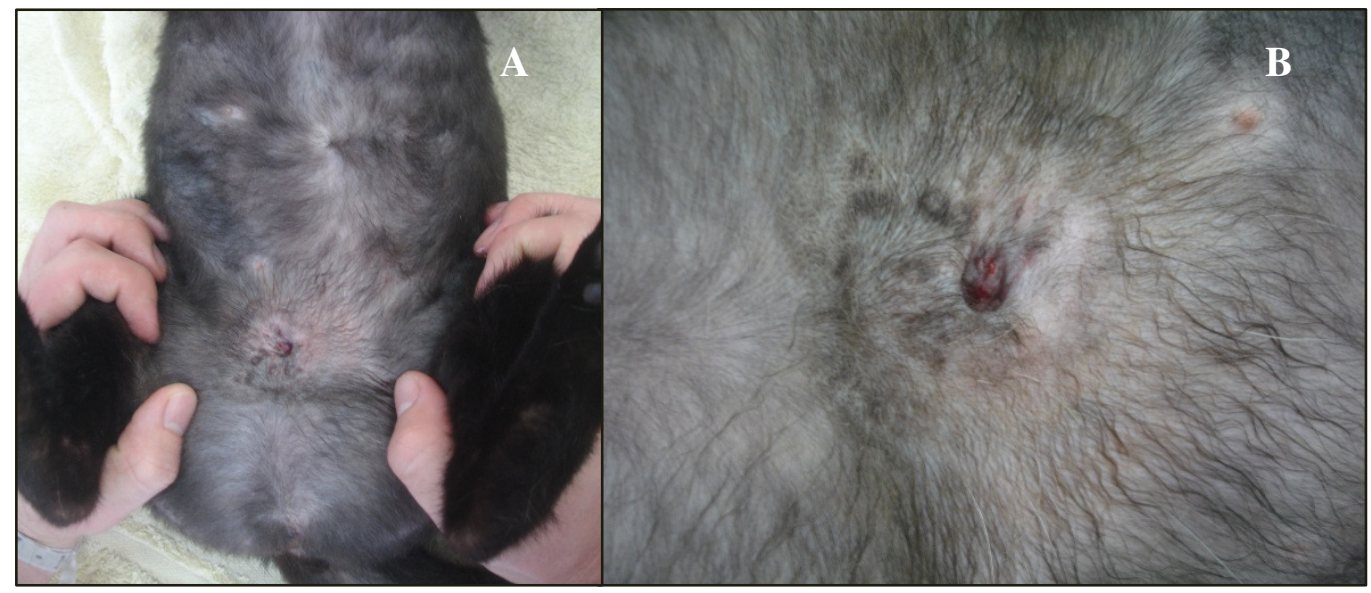

Fonte: Elaboração dos autores.

A DTUIF acomete frequentemente felinos domésticos, causando alterações vesicais e uretrais, caracterizadas por hematúria, disúria, polaciúria e obstrução uretral parcial ou completa (ROSA; QUITZAN, 2011). O paciente em questão apresentava histórico de DTUIF recorrente e já tinha sofrido cinco cirurgias desde aproximadamente seus três anos de idade para correção da enfermidade. Havia passado por uma uretrostomia perineal e mais quatro intervenções subsequentes na tentativa de corrigir a primeira, sem obtenção de função aceitável.

A uretrostomia pré-púbica é uma técnica indicada em obstruções graves e em processos de estenose da uretra perineal ou peniana. $\mathrm{O}$ caso em questão foi considerado grave, já que o paciente encontravase totalmente obstruído e a sondagem uretral não foi possível. A escolha da técnica também deveu-se ao histórico de repetidas tentativas cirúrgicas para desobstrução uretral. Porém, apesar das vantagens na utilização, tal procedimento poderá predispor a infecção do trato urinário e apresenta risco mínimo de estenose (PINTO, 2011; BERNARDE; VIGUIER, 2004), o que não foi observado até o momento no paciente em questão.

Até o período de oito meses de pós-operatório não foi verificada a ocorrência de estenose uretral, sendo que o animal também não apresentou incontinência urinária. Porém, há alguns dias apresentou hematúria e foi tratado, sob orientação veterinária, com enrofloxacina $(5 \mathrm{mg} / \mathrm{kg}, \mathrm{VO}, \mathrm{SID}$ durante sete dias) e meloxicam $(0,1 \mathrm{mg} / \mathrm{kg}, \mathrm{VO}, \mathrm{SID}$ por três dias). Segundo a proprietária, nas primeiras 24 horas de tratamento já houve desaparecimento da hematúria. Esta foi a primeira vez que o animal apresentou sinais de infecção do trato urinário 
inferior após o procedimento cirúrgico. Após o tratamento a proprietária retornou ao hospital com o felino, o qual já demonstrava completa recuperação, não apresentando mais hematúria ao exame clínico. $\mathrm{Na}$ oportunidade, foi verificada presença de dermatite ao redor da abertura da uretrostomia, para qual se recomendou limpeza com solução de $\mathrm{NaCl}$ $0,9 \%$ duas vezes por semana e remoção dos pêlos da área a fim de evitar o acumulo de urina.

No caso relatado, utilizou-se uma variação da técnica previamente proposta (PINTO, 2011). Este autor realizou a técnica reparando e seccionando a uretra no sentido transversal, obliterando a extremidade distal remanescente com fios de sutura e pontos isolados simples. A seguir, suturou a mucosa da extremidade uretral proximal com a pele através de náilon 4-0 em padrão isolado simples, concluindo o óstio uretral. No relato em questão, dissecou-se apenas a face uretral dorsal, objetivando lesionar o mínimo a vascularização e inervação locais, posteriormente seccionou-se a uretra longitudinalmente e, a seguir, a mucosa foi suturada a pele com polidioxanona 5-0 em padrão isolado simples. Assim, manteve-se os seus princípios, como método de salvamento para o felino, o qual já havia passado por vários procedimentos de uretrostomia perineal e correções subsequentes. Obteve-se sucesso terapêutico, possibilitando ao paciente adequada qualidade de vida.

Conclui-se que a uretrostomia pré-púbica laparoscópica híbrida é factível e segura para gatos, tornando-se uma opção adequada de tratamento cirúrgico para estenoses uretrais graves.

\section{Referências}

BAINES, S. J.; RENNIE, S.; WHITE, S. A. Prepubic urethrostomy: a long-term study in 16 cats. Veterinary Surgery, Davis, v. 30, n. 2, p. 107-113, 2001.

BERNARDE, A.; VIGUIER, E. Transpelvic urethrostomy in 11 cats using an ischial ostectomy. Veterinary Surgery, Davis, v. 33, n. 3, p. 246-252, 2004.

GALVÃO, A. L. B.; ONDANI, A. C.; FRAZÍLIO, F. O.; FERREIRA, G. S. Obstrução uretral em gatos machos revisão literária. Acta Veterinaria Brasilica, Mossoró, v. 4, n. 1, p. 1-6, 2010.

KAUFMANN, C.; NEVES, R. C.; HABERMANN, J. C. A. Doença do trato urinário inferior dos felinos. Anuário da Produção Científica dos Cursos de Pós-Graduação, v. 4, n. 4, p. 193-214, 2009.

LEÓN, J. C.; GILL, M. S.; CORNICK-SEAHORN, J. L.; HEDLUND, C. S.; HOSGOOD, G. Prepubic urethrostomy for permanent urinary diversion in two Vietnamese pot-bellied pigs. Journal of the American Veterinary Medical Association, New York, v. 210, n. 3, p. 366-368, 1997.

PINTO, T. M. Uretrostomia pré-púbica por laparoscopia vídeo-assistida: modelo experimental em coelhos. 2011. Dissertação (Mestrado em Ciências Veterinárias) Programa de Pós-graduação em Ciências Veterinárias. Universidade Federal do Rio Grande do Sul, Porto Alegre.

ROSA, V. M.; QUITZAN, J. G. Avaliação retrospectiva das variáveis etiológicas e clínicas envolvidas na doença do trato urinário inferior dos felinos (DTUIF). Cesumar, Maringá, v. 13, n. 2, p. 103-110, 2011. 
